
\title{
Resesach S Surure \\ Research an Online Monitoring Method of Propulsion Shafting Alignment Adapting to Hull Deformation
}

Jianwei Cheng

Naval University of Engineering

Liang Shi

Naval University of Engineering

Yuanchao Zhang

Naval University of Engineering

Wenjun Bu ( $\nabla$ bwj2005@163.com )

Naval University of Engineering

\section{Research Article}

Keywords: hull deformation, propulsion shafting, Euler rigid body attitude, online shafting alignment monitoring

Posted Date: September 23rd, 2021

DOI: https://doi.org/10.21203/rs.3.rs-900092/v1

License: (c) (i) This work is licensed under a Creative Commons Attribution 4.0 International License. Read Full License 


\title{
Research an Online Monitoring Method of Propulsion Shafting Alignment Adapting to Hull Deformation
}

\author{
Jianwei Cheng ${ }^{1,2}$, Liang Shi ${ }^{1,2}$, Yuanchao Zhang ${ }^{1,2} \&$ Wenjun Bu ${ }^{1,2, *}$ \\ 1.Institute of Noise \& Vibration, Naval University of Engineering, Wuhan, China, \\ 2.National Key Laboratory on Ship Vibration \& Noise, Hubei, China \\ *Corresponding author: WenJun Bu, bwj_2005@163.com
}

\begin{abstract}
Hull deformations are caused by factors such as loading status, wave load, diving depth of underwater vehicles, etc. This causes a change in the shafting alignment state of the ship's propulsion shafting. This affects the alignment state of the ship propulsion shafting. It is very important for engineering significance to research an online monitoring method of propulsion shafting alignment that adapts to the influence of hull deformation. Based on Euler's rigid body attitude theory, this paper constructs a spatial attitude relationship model of the main and driven shafts of the ship's propulsion shafting. By converting to a fixed earth coordinate system, their absolute position-posture can be obtained. Using laser displacement sensor measurement technology, an online monitoring method of propulsion shafting alignment that adapts to the influence of hull deformation is established, which was verified by experiments. This method is suitable for situations where both the main shaft and the driven shaft may have rigid body posture changes. It can provide shafting alignment control compensation for the influence of hull deformation under different working conditions. This will greatly reduce the operating noise of the shaft system and the mechanical operation failure caused by the misalignment of the ship's propulsion shafting.
\end{abstract}

Keywords: hull deformation ; propulsion shafting ; Euler rigid body attitude ; online shafting alignment monitoring

Ship propulsion shafting is an important component for transmitting the output torque of the main engine.Shafting misalignment will produce a pulsating excitation force on the shafting system, resulting in radial vibration and axial vibration[1].By analyzing the motion equation of an ordinary rotor bearing system, $\mathrm{Zw}$ et al. [2] found that as the degree of shaft misalignment increases, the average value of rotor radial vibration increases significantly.Shafting misalignment will affect the stress of the shaft, the pressure distribution and the reaction force of the bearing. Shafting misalignment changes the lateral and torsional vibration characteristics of the the shafting[3].Shafting misalignment is divided into parallel misalignment(offset), angular misalignment(skew) and combined misalignment (deviation, including parallel misalignment and angular misalignment). The influence of parallel misalignment is dominant at 2 times the characteristic frequency[4].Parallel misalignment shows a stronger $1 \mathrm{x}$ axial and torsional response, and the angular misalignment shows a very strong $3 \mathrm{x}$ harmonic component in the axial and torsional vibration response at $1 / 3$ of the critical speed [5].The uncertainty of parallel misalignment affects odd frequency components, while angular misalignment affects even frequency components [6].Therefore, shafting misalignment will increase the vibration and noise of the shafting operation. In severe cases, it may cause operation failure of the propulsion system.According to the literature, more than $70 \%$ of mechanical failures of rotating machinery are caused by misalignment[7].

In terms of shaft alignment, ship propulsion is very different from land-based rotating machinery. The installation foundation of the ship's propulsion shafting is the hull.The loading status, wave load, etc.will cause the deformation of the hull.Especially for underwater vehicles, diving depth will also produce hull deformations. However, land-based rotating machinery usually does not have such deformation problems.Under different working conditions, the hull deformation changes may worsen the alignment state of the propulsion shafting.In severe cases, it may affect the operational safety of the ship's propulsion system. Ruan et al. [8] took an 8,530 TEU container ship as the research object and calculated the deformation of the hull at the shaft support under different loads and waves through the FEM.The hull deformation at the propulsion shaft support was as high as $10 \mathrm{~mm}$. Therefore, it is necessary to consider the influence of hull deformation when studying the alignment state of the ship's propulsion shafting.It is of great engineering significance to research an online monitoring method of propulsion shafting alignment and to realize the online monitoring of its operating status, which adapts to hull deformation. 
The existing shafting alignment measurement technology is basically offline, which is only suitable for shafting installation inspection and measurement. The more common ones are the dial indicator method and the laser alignment instrument method.Garg et al.[9] and $\mathrm{Li}$ and Wang [10] used two laser sensors (PSD/LD-PSD, Figure 1(a) and Figure 1(b)) to collect data from three different positions of the laser sensor to calculate the shaft alignment state. The two laser sensors are respectively installed at the main and driven ends of the propulsion shaft system, and the sensor is rotated with the shaft system by cranking the shaft system. These methods are suitable for the nonworking state of the shaft system. Once the shaft system is working normally, the laser sensor cannot be rotated for measure.Therefore, it is impossible to realize the online monitoring of the shaft alignment state, and it is not suitable for shaft alignment measurement under the condition of the deformation of the hull.

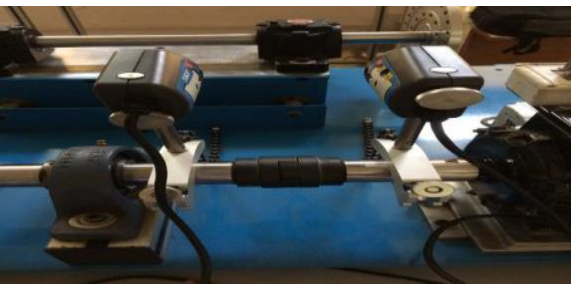

(a) Structure of the laser alignment system with dual PSDs [9]

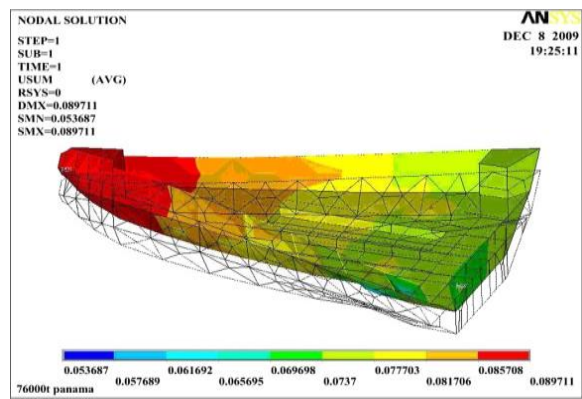

(c) An example of ship hull FEM model with hull deformations[13]

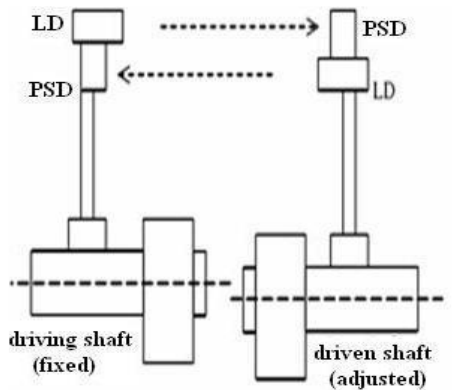

(b) Two-beam LD/PSD diagram[10]

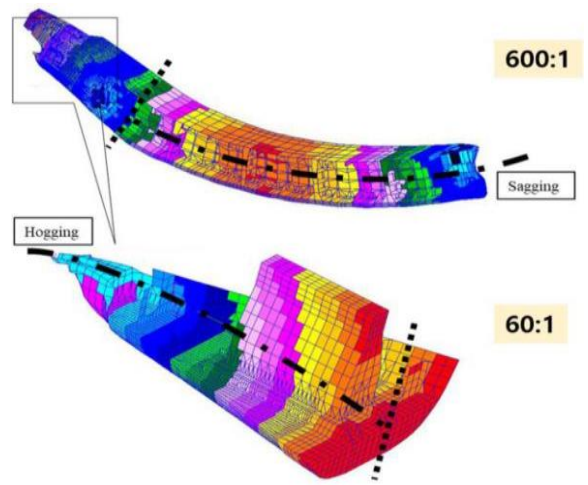

(d) Hull deformation at St5

(Full draught APT empty) condition[15]

Figure 1. Monitoring principle and hull deformation

Ttraditional reasonable shaft alignment technology[11] ignores dynamic factors such as hull deformation. Dynamic shaft alignment technology is still in the stage of theoretical research, which considers hull deformation and other dynamic factors. The dynamic alignment technology is closer to the actual working conditions of the shaft system, which can better improve the shaft alignment quality and the reliability of the shaft system operation. Shi Lei et al.[12][13], Li Zeyuan et al.[14] and Seo et al.[15] used the FEM to research the relationship between hull deformation and bearing displacement. Shi Lei [13] took a 76,000 DWT product oil tanker as the research object.Hull deformation (Figure 1(c)) is analyzed by an FEM, and the deformation of the bottom hull is transformed into the deformation of the bearing.Compared with the standard line, the bearing offset is estimated.Compared with the azimuth offset in the still water condition, the relative azimuth offset is estimated. Seo et al.[15] took the 300,000 DWT ultralarge crude oil carrier as the research object. According to the ship's draft, five draft conditions were selected to analyze the hull deformation of the overall structure and calculate the bearing offset value. The analysis results (Figure 1(d)) show that the hull deformation may be a key factor affecting the deflection and deformation of each bearing supporting the shafting.Generally, shipyards use the FEM to analyze the influence of hull deformation on the vertical relative deformation of the shafting centerline under floating conditions, ballast conditions and full load conditions to optimize the shaft alignment design.Although the influence of hull deformation on the position offset of the shafting bearing is researched, there is a lack of research on the calculation of parallel misalignment and angular misalignment, and it is impossible to realize the online monitoring of the system's centering state. 
Zw et al. [2] used three displacement sensors to monitor diesel engine misalignment, and realized real-time calculation of the misalignment. Two parallel sensors with a fixed relative position quantitatively detect the posture of the output shaft. The relative position of the two sensors will change under the influence of the hull deformation. $\mathrm{Bu}$ Wenjun et al. [16] (Figure 2 is the schematic diagram) and Shi Liang et al. [17] used an eddy current displacement sensor to measure the horizontal and vertical displacements of different parts of the propulsion main engine to construct an online shaft alignment monitoring model. In this way, based on the hull (assuming that there is no deformation in the hull), the online alignment monitoring of the propulsion main engine shafting system is realized. Applied to the smart air spring vibration isolation device, the maintaining control of the shafting attitude of the ship propulsion main engine is realized [16-19].However, these methods do not take into account the impact of hull deformation. They cannot adapt to the impact of hull deformation to achieve accurate online monitoring of shaft alignment. There are two main problems. On the one hand, these methods assume that the driven shaft does not perform any rigid movement. The parallel misalignment and angular misalignment of the main shaft relative to the oxy plane are the alignment values of the shaft system. The hull deformation will affect the position offset of the main shaft and the driven shaft. Therefore, the alignment value is determined by the position states of both the main shaft and the driven shaft at the same time. On the other hand, with the development of floating raft vibration isolation technology, raft bodies are carrying increasing equipment, and raft bodies are developing toward larger and lighter weight [20]. The deformation of the elastic support platform affects the shafting deformation [21].The measurement data of the displacement sensor include the local deformation of the shaft installation platform, which cannot be simply regarded as the pure rigid displacement of the main engine.

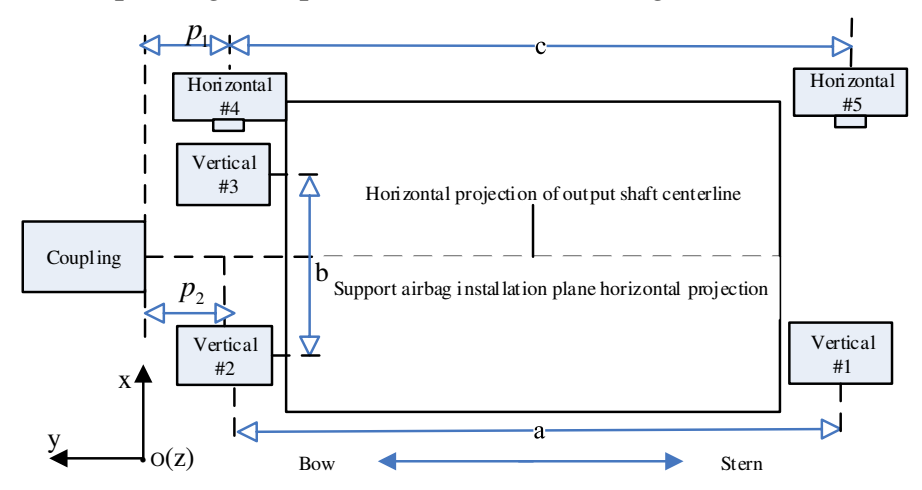

Figure 2. Schematic diagram of the displacement sensor layout of the alignment attitude monitoring system [16]

Based on Euler's rigid body attitude theory, this paper constructs the space position-posture relationship model of the main shafts and driven shafts of the ship's propulsion shaft system. Their relative space position-posture is converted into the absolute position-posture state of the Earth's fixed coordinate system. Using the measurement technology of laser displacement sensor, an online propulsion shafting that can adapt to the influence of hull deformation is established, and the method is tested and verified.

\section{Principle of the online alignment monitoring method for propulsion shafting}

The hull deformation causes position changes of the propulsion motors and bearings. Both the main shaft and the driven shaft will have different rigid body motions and produce displacements of different amplitudes. Therefore, when researching shaft alignment, it is necessary to consider the position and position states of both the main shaft and the driven shaft at the same time. As shown in Figure 3, the relative position-posture of the main shaft and the driven shaft is obtained by two laser sensors. They are respectively fixed on the main and driven shaft fixing brackets. By analyzing the position and position data of the two shafts, the position and position change of the two shafts is obtained, and then the shafting alignment value is calculated. Therefore, the shafting online alignment monitoring system was constructed. The workflow is shown in Figure 4. 


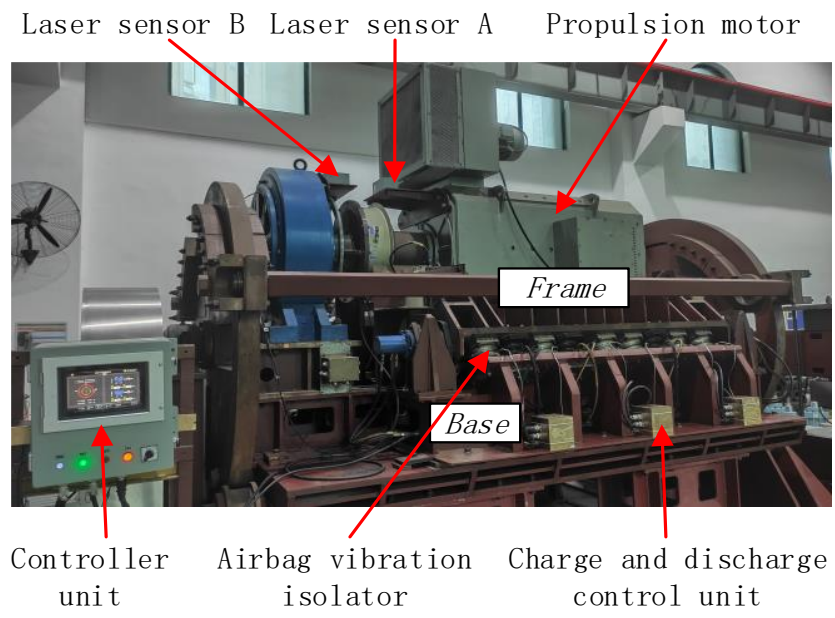

Figure 3. The relative position-posture of the main shaft and the driven shaft obtained by two laser sensors

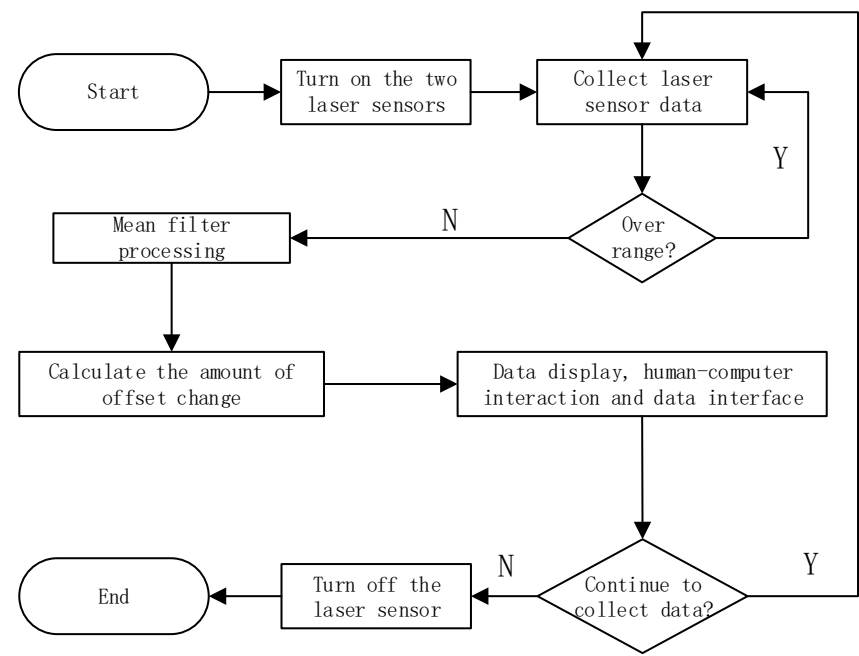

Figure 4. The workflow of the shafting online alignment monitoring system

This system is mainly composed of a laser displacement sensor, relay bridge module and other modules. Among them, laser sensor A and laser sensor B are used to measure the relative position-posture of the driven shaft and main shaft respectively.The relay bridge module is responsible for controlling the opening and closing of the laser sensor, collecting the laser sensor data and judging whether it is out of range, capturing multiple sets of data for average filtering, and using the centering offset model to calculate the centering change and display it to the user. At the same time, users can set up and perform corresponding data processing and display functions.

\section{Alignment monitoring model}

As shown in Figure 5, three coordinate systems are established: a fixed spatial reference coordinate system, a body A coordinate system and a body $\mathrm{B}$ coordinate system. $o_{-} x y z$ represents the earth fixed reference coordinate system. $O_{-} X_{A} Y_{A} Z_{A}$ represents the A body coordinate system fixedly connected to the center point of the laser sensor A. $O_{-} X_{B} Y_{B} Z_{B}$ represents the $\mathrm{B}$ body coordinate system fixedly connected to the center point of the laser sensor B. $\psi_{A}$ represents the torsion angle of the laser sensor A. $\psi_{B}$ represents the laser sensor the torsion angle of B. The coordinate of point $\mathrm{P}$, one point on the coupling, is $\left(X_{P}, Y_{P}, Z_{P}\right)$ in the $O_{-} X_{A} Y_{A} Z_{A}$ coordinate system . The $O_{-} X_{A} Y_{A} Z_{A}$ and $O_{-} X_{B} Y_{B} Z_{B}$ coordinate systems are the same as the azimuth, and the initial state (state 1 ) has a different torsion angle $\psi_{0}\left(\psi_{0}=\psi_{A 1}-\psi_{B 1}\right)$. 


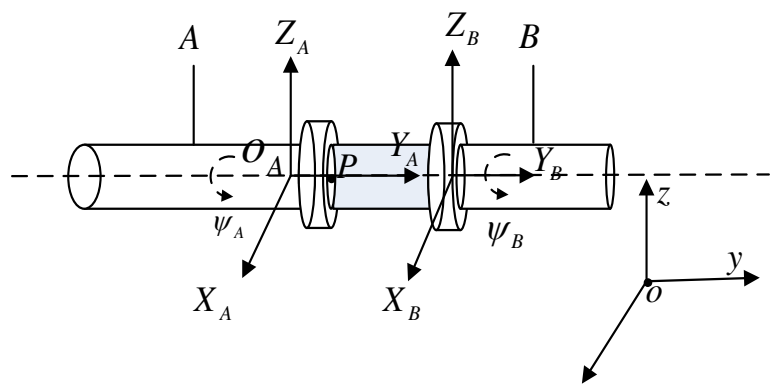

Figure 5. Schematic diagram of the ship propulsion shaft system and coordinate system

For any point $Q\left(x_{Q}, y_{Q}, z_{Q}\right)$ on the $O_{-} X_{A} Y_{A} Z_{A}$ coordinate system, since $O_{-} X_{A} Y_{A} Z_{A}$ rotates $\psi_{A}$ around the $o y$ axis relative to $o_{-} x y z$, the coordinates of point $Q$ in the coordinate system $o_{-} x y z$ are:

$$
\left[\begin{array}{lll}
x & y & z
\end{array}\right]^{T}=R_{Y}\left(\psi_{A}\right) \cdot\left[\begin{array}{lll}
x_{Q} & y_{Q} & z_{Q}
\end{array}\right]^{T},
$$

where $\mathrm{R}_{\mathrm{A}}\left(\psi_{\mathrm{A}}\right)$ is the rotation transformation matrix:

$$
R_{Y}\left(\psi_{A}\right)=\left[\begin{array}{ccc}
\cos \psi_{A} & 0 & \sin \psi_{A} \\
0 & 1 & 0 \\
-\sin \psi_{A} & 0 & \cos \psi_{A}
\end{array}\right],
$$

In this article, it assumed that the main shaft and driven shaft do not undergo elastic deformation. In the main shaft and driven shaft only rigid body motion occurs. The position-posture of laser sensors A and B on the main shaft and driven shaft can be used to deduce the position-posture of the entire shaft system through the law of rigid body position-posture movement.The Laser sensors A and B emit laser beams opposite to each other along the oy axis, and form imaging points on each other. As shown in Figure 6, the torsion angles of Laser sensor A and B are $\psi_{A 1}$ and $\psi_{B 1}$ in state 1 respectively, and are $\psi_{A 2}$ and $\psi_{B 2}$ in state 2 .

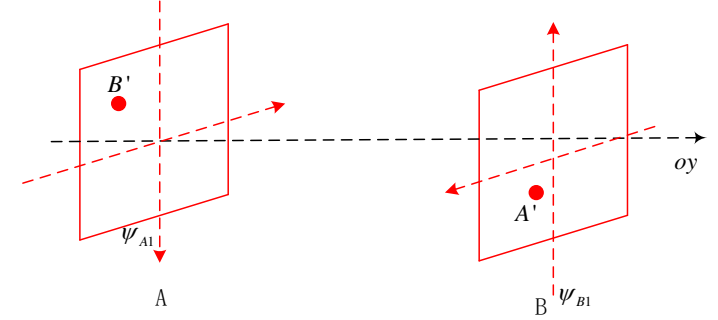

(a) state 1

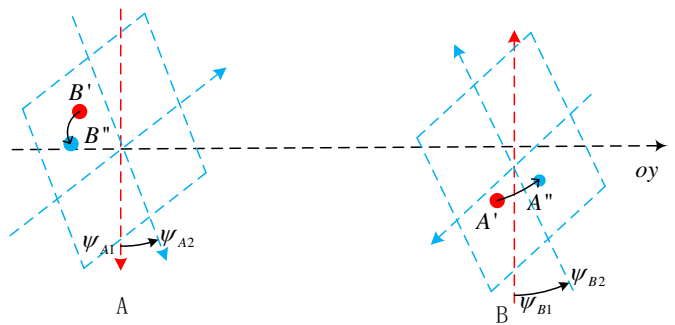

(b) state 2

Figure 6. Schematic diagram of monitoring model

The imaging points of laser sensors $A$ and $B$ in state 1 are $B^{\prime}\left(X_{A 1}, 0, Z_{A 1}\right)$ and $A^{\prime}\left(X_{B 1}, 0, Z_{B 1}\right)$, and the imaging points of laser sensors $\mathrm{A}$ and $\mathrm{B}$ in state 2 are $B^{\prime \prime}\left(X_{A 2}, 0, Z_{A 2}\right)$ and $A^{\prime \prime}\left(X_{B 2}, 0, Z_{B 2}\right)$. Since laser sensor $\mathrm{A}$ is rotated $180^{\circ}$ relative to $O_{-} X_{A} Y_{A} Z_{A}$, in the $O_{-} X_{A} Y_{A} Z_{A}$ coordinate system, the coordinates of $A^{\prime}$ and $A^{\prime \prime}$ are $\left(-X_{B 1}, 0,-Z_{B 1}\right)$ and $\left(-X_{B 2}, 0,-Z_{B 2}\right)$.Therefore, transformed to the $o_{-} x y z$ coordinate system, the changes in the $o x$ direction and $O Z$ direction between the imaging point in state 1 and the imaging point in state 2 are:

$$
\begin{aligned}
& {\left[\begin{array}{c}
\Delta x_{A} \\
0 \\
\Delta z_{A}
\end{array}\right]=R_{Y}\left(\psi_{B 2}\right)\left[\begin{array}{c}
X_{A 2} \\
0 \\
Z_{A 2}
\end{array}\right]-R_{Y}\left(\psi_{B 1}\right)\left[\begin{array}{c}
X_{A 1} \\
0 \\
Z_{A 1}
\end{array}\right],} \\
& {\left[\begin{array}{c}
\Delta x_{B} \\
0 \\
\Delta z_{B}
\end{array}\right]=-R_{Y}\left(\psi_{A 2}\right)\left[\begin{array}{c}
X_{B 2} \\
0 \\
Z_{B 2}
\end{array}\right]+R_{Y}\left(\psi_{A 1}\right)\left[\begin{array}{c}
X_{B 1} \\
0 \\
Z_{B 1}
\end{array}\right],}
\end{aligned}
$$

For $\psi_{A 1}, \psi_{B 1}, \psi_{A 2}$ and $\psi_{B 2}$ are all small angle angles, so Eqs. 3-4 can be simplified to:

$\Delta x_{A}=\left[X_{A 2} \cos \left(\psi_{0}+\Delta \psi\right)+Z_{A 2} \sin \left(\psi_{0}+\Delta \psi\right)\right]-\left(X_{A 1} \cos \psi_{0}+Z_{A 1} \sin \psi_{0}\right)$,

$\Delta z_{A}=\left[-X_{A 2} \sin \left(\psi_{0}+\Delta \psi\right)+Z_{A 2} \cos \left(\psi_{0}+\Delta \psi\right)\right]-\left(-X_{A 1} \sin \psi_{0}+Z_{A 1} \cos \psi_{0}\right)$,

$\Delta x_{B}=-\left[X_{B 2} \cos \left(\psi_{0}+\Delta \psi\right)+Z_{B 2} \sin \left(\psi_{0}+\Delta \psi\right)\right]+\left(X_{B 1} \cos \psi_{0}+Z_{B 1} \sin \psi_{0}\right)$,

$\Delta z_{B}=-\left[-X_{B 2} \sin \left(\psi_{0}+\Delta \psi\right)+Z_{B 2} \cos \left(\psi_{0}+\Delta \psi\right)\right]+\left(-X_{B 1} \sin \psi_{0}+Z_{B 1} \cos \psi_{0}\right)$, 
Eqs. 5-6 are the position-posture changes of the driven shaft. Eqs. 7-8 are the position-posture changes of the main shaft.Generally, parallel misalignment(offset deviation) and angular misalignment(skew deviation) are used to measure the shaft alignment state. The shaft alignment state response vector [22] is:

$$
A=\left[\begin{array}{llllll}
\Delta x_{c} & \Delta y_{c} & \Delta z_{c} & \theta & \varphi & \psi
\end{array}\right]^{T},
$$

In this article, the axial deformation $\Delta y_{c}$ is not considered. The torsion angle $\psi$ can be measured by the inclination sensor. The coupling point $P$ is selected as the calculation point of the alignment state change. The alignment state change of any two states is:

$$
A=\left[\begin{array}{llll}
\Delta x_{P} & \Delta z_{P} & \Delta \theta & \Delta \varphi
\end{array}\right]^{T},
$$

Eqs. 5-8 calculate the position-posture change of the main shaft and driven shaft. Because the horizontal angularity change $\Delta \theta$ and the vertical angularity change $\Delta \varphi$ are both small angles, they can be approximated as:

$$
\Delta \theta=\tan \Delta \theta, \Delta \varphi=\tan \Delta \varphi
$$

so there is a geometric relationship:

$$
\begin{aligned}
& \Delta \theta=\frac{\Delta x_{B}-\Delta x_{A}}{b}, \\
& \Delta \varphi=\frac{\Delta z_{A}-\Delta z_{B}}{b},
\end{aligned}
$$

where $\mathrm{b}$ is the axial center distance of the two optical instruments.

The horizontal offset change $\Delta x_{P}$ and the vertical offset change $\Delta z_{P}$ are:

$$
\begin{aligned}
& \Delta x_{P}=\Delta x_{B}-\Delta \theta \cdot Y_{P}+\Delta \psi \cdot Z_{P}, \\
& \Delta z_{P}=\Delta z_{\mathrm{B}}+\Delta \varphi \cdot Y_{P}-\Delta \psi \cdot X_{P},
\end{aligned}
$$

Eqs. 11-14 are the calculation models for the amount of change in the alignment component. In particular, when state 1 is the ideal shaft alignment state, the model is the shaft alignment component calculation model.

\section{Experimental verification}

A comprehensive experimental platform for shaft alignment of the main power propulsion system is built. The physical and schematic diagram are shown in Figure 7.
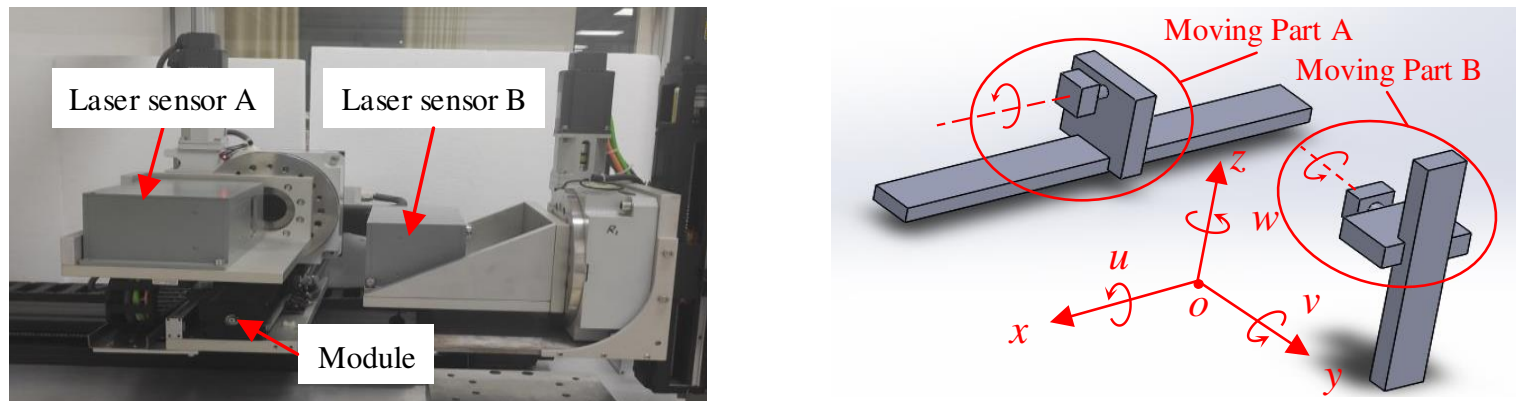

(b) Schematic diagram

(a) Physical image

Figure 7. Experimental platform

The Laser sensor A is fixed on the moving Part A of the experimental platform. The Laser sensor B is fixed on the moving Part B of the experimental platform. Rigid body motion has 6 freedom degrees. In this article, the amount of axial deformation (oy component) is not considered.The position-posture motions of the main shaft and driven shaft of the shaft system are relative. To reduce the number of variables, when studying the relative positionposture relationship between the main shaft and driven shaft, it can be considered that the main shaft has two degrees of freedom ( $o x$ and $o u$ ), and the driven shaft has the other two degrees of freedom( $O Z$ and $o v$ ). Moving Part A simulates the position-posture state of the main shaft, which has two degrees of freedom. It can realize the horizontal parallel misalignment component of the shaft system through translational movement along the $o x$ direction. At the same time, it can make the rotation movement around the $o x$ axis $(o u)$ to realize the vertical angular misalignment of the shaft system.Moving Part B simulates the position-posture state of the driven shaft, which has two degrees of freedom. It can realize the vertical parallel misalignment component of the shaft system through translational movement along the $O Z$ direction. In addition, it can make the rotation movement of around the $o y$ axis $(\mathrm{ov})$ to realize the torsional rotation of the shaft system. Therefore, by controlling the translation and rotation of the A and B components, different alignment states of the shaft system can be achieved, including horizontal 
parallel misalignment, vertical parallel misalignment and vertical angular misalignment. The main parameters of the experimental platform are shown in Table 1.

Table 1. Parameters list of the experimental platform

\begin{tabular}{|c|c|c|}
\hline & unit & description \\
\hline \multirow{3}{*}{ translational parts } & motion drive unit & using Siemens V90 series $400 \mathrm{~W}$ AC $220 \mathrm{~V}$ servo motor \\
\hline & $\begin{array}{l}\text { motion module unit } \\
\quad \text { (slide rail) }\end{array}$ & $\begin{array}{l}\text { using KK86 series, length: } 340 \mathrm{~mm} \text {, maximum sliding stroke: } \\
\text { 210mm, positioning reproduction accuracy: } \pm 0.003 \mathrm{~mm}, \\
\text { positioning accuracy: } 0.025 \mathrm{~mm} \text {, movement parallelism : } 0.015 \\
\mathrm{~mm}\end{array}$ \\
\hline & $\begin{array}{l}\text { displacement } \\
\text { feedback unit }\end{array}$ & $\begin{array}{l}\text { using grating ruler, resolution: } 5 \mu \mathrm{m}, \text { positioning accuracy: } \leq 10 \\
\mu \mathrm{m}\end{array}$ \\
\hline \multirow{3}{*}{ rotating parts } & motion drive unit & Siemens V90 series $400 \mathrm{~W}$ AC $220 \mathrm{~V}$ servo motor \\
\hline & index plate unit & $\begin{array}{l}\text { using the servo motor to driveERT200-A1 index plate to rotate, } \\
\text { repeating positioning accuracy:0.003 }{ }^{\circ} \text {, ratio: } 88(1: 44), \\
\text { concentricity : } 30 \mu\end{array}$ \\
\hline & angle feedback unit & $\begin{array}{l}\text { using ACT926T-5 high-precision tilt sensor, measurement range: - } \\
5^{\circ} \sim+5^{\circ} \text {, zero temperature drift: } \pm 0.0005^{\circ}\left(-40^{\circ} \mathrm{C} \sim 85^{\circ} \mathrm{C}\right) \text {, } \\
\text { resolution: } 0.0005^{\circ} \text {, accuracy: } 0.001^{\circ} \text {, response time: } 0.01 \mathrm{~s}\end{array}$ \\
\hline
\end{tabular}

The translation accuracy of Moving Parts A (along the $o x$ axis) and B (along the $o z$ axis)is $0.01 \mathrm{~mm}$, and the moving range is $-15+15 \mathrm{~mm}$. The rotation accuracy of Moving Parts A (around the $o x$ axis) and B (around the $o y$ axis) is $0.005^{\circ}(0.0875 \mathrm{~mm} / \mathrm{m})$, and the rotation range is $-1^{\circ} \sim+1^{\circ}$.

The main parameters of the laser sensor are as follows. The maximum transmitting and receiving distance is 20 $\mathrm{m}$. The receiver range is $-8 \sim 8 \mathrm{~mm}$. The receiver resolution is $0.001 \mathrm{~mm}$. The linear error is $1 \% \pm 0.003 \mathrm{~mm}$. The working temperature range is $-5^{\circ} \mathrm{C} \sim 50^{\circ} \mathrm{C}$. The signal output mode is the $\mathrm{RS} 485$ protocol .

To verify the correctness of the shafting alignment model that adapts to the deformation of the hull, three types of targeted tests have been carried out, namely, the purely parallel misalignment condition, the purely angular misalignment condition and the combined misalignment condition test[23][24].Three types of centering components of horizontal offset, vertical offset and vertical angularity are simulated by controlling the movement of Moving Parts A and B. The offset and angularity of the control movement are recorded. At the same time, using the two-axis relative position-posture obtained by the laser sensor, the corresponding three centering components are calculated. Thus the difference between the control centering component and the solution centering component is calculated, which is Horizontal Offset Error (HOE), Vertical Offset Error (VOE) and Vertical Angularity Error (VAE). So the offset error (Offset Error, OE) is the arithmetic square root of the horizontal offset error and the vertical offset error $\left(O E=\sqrt{H O E^{2}+V O E^{2}}\right)$. Then an error map will be drawn and the root mean square error will be calculated to evaluate the accuracy of the calculation model.
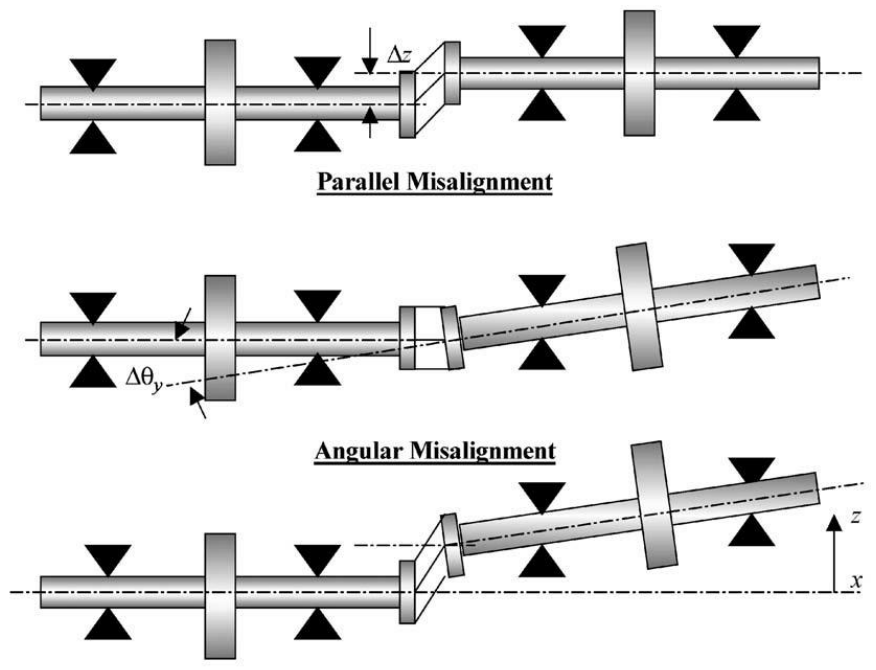

Combined Misalignment

Figure 8. Schematic of rotor with misalignment at a coupling[24] 
Aiming at the three types of shafting misalignment including parallel misalignment, angular misalignment and combined misalignment [24] (Figure 8), three types of experiments were designed and carried out in purely parallel misalignment conditions, purely angular misalignment conditions and combined misalignment conditions [23]. A total of 6 sets of experiments were carried out. Experiments \#1 to \#3 are used to verify the purely parallel conditions, corresponding to vertical translation, horizontal translation and combined translation, respectively, and corresponding to verifying vertical offset, horizontal offset and horizontal and vertical offset. Experiment \#4 is used to verify pure rotation conditions, corresponding to pitch rotation, and is used to verify vertical deflection. Experiment \#5 and Experiment \#6 are used to verify the combined parallel-rotation working conditions, corresponding to horizontal translation-pitch rotation and vertical translation-pitch rotation, corresponding to verifying horizontal offset-vertical skew deviation and horizontal offset-vertical straight skew error.The working conditions are shown in Table 2.

Table 2. List of working conditions

\begin{tabular}{|c|c|c|l|}
\hline motion conditions & No. & verification type & \multicolumn{1}{c|}{ realization } \\
\hline \multirow{3}{*}{ purely parallel } & $\# 1$ & vertical offset & $\begin{array}{l}\text { control the sliding movement of Moving } \\
\text { Part B along the } o z \text { axis }\end{array}$ \\
\cline { 2 - 5 } & $\# 2$ & horizontal offset & $\begin{array}{l}\text { control the sliding movement of Moving } \\
\text { Part A along the } o x \text { axis }\end{array}$ \\
\hline \multirow{2}{*}{$\begin{array}{c}\text { pure rotation } \\
\text { parallel-rotation } \\
\text { combination }\end{array}$} & $\# 4$ & horizontal-vertical offset & $\begin{array}{l}\text { control Moving Part A to slide along the } \\
o x \text { axis, and control Moving Part B to } \\
\text { slide along the } o z \text { axis }\end{array}$ \\
\cline { 2 - 5 } & $\# 5$ & vertical angularity & $\begin{array}{l}\text { control the rotating movement of } \\
\text { Moving Part A around the } o x \text { axis }\end{array}$ \\
\hline (horizontal offset and vertical angularity) & $\begin{array}{l}\text { control Moving Part A to slide along the } \\
\text { ox axis, and control Moving Part A to } \\
\text { rotate around the } o x \text { axis }\end{array}$ \\
\hline
\end{tabular}

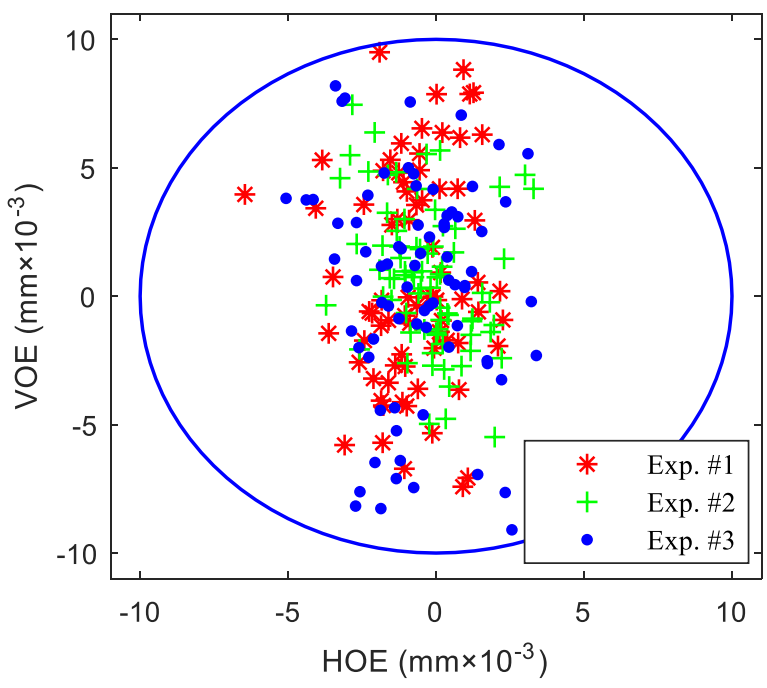

(a) The deviation error diagram of \#1 \#3 experiment

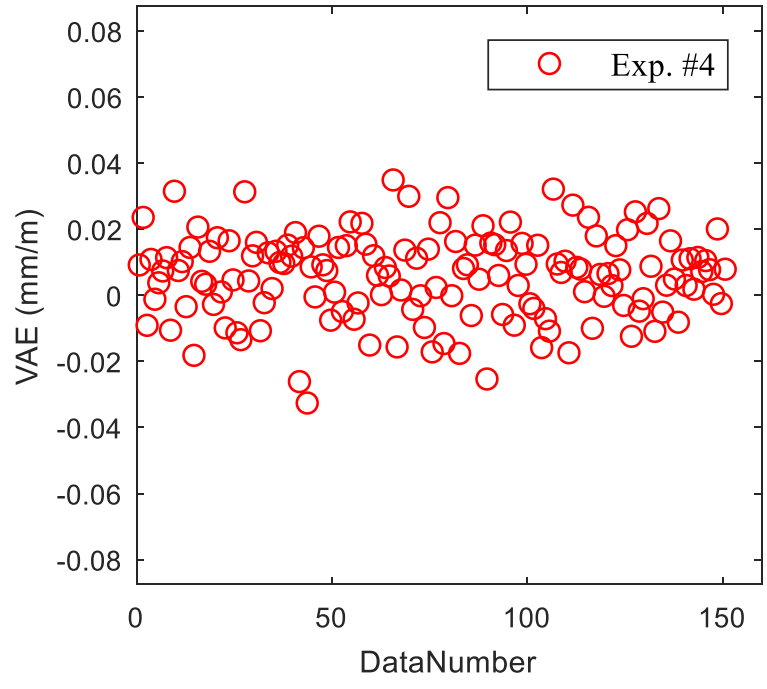

(b) The deflection error diagram of \#4 experiment

Figure 9. Error diagram of parallel and rotation

Experiments \#1 to \#3 were carried out for purely parallel motion conditions. From the experimental results (Figure 9a), it can be seen that the horizontal offset error presents a clustered distribution, and the vertical offset error distribution is relatively scattered. The offset errors $(\mathrm{OE})$ are all less than $0.01 \mathrm{~mm}$. Considering the parallel accuracy of the experimental platform $(0.01 \mathrm{~mm})$, for the axis system offset state, the offset calculation model for the change component of the shaft alignment state is accurate. Experiment \#4 was carried out founder pure rotation conditions. From the experimental results (Figure 9b), it can be seen that the vertical angularity error is concentrated 
in the range of $-0.02 \sim 0.02 \mathrm{~mm} / \mathrm{m}$. The results of pure translation and pure rotation RMSE are shown in Table 3 . Considering the rotation accuracy of the experimental platform $(0.0875 \mathrm{~mm} / \mathrm{m})$, the angularity calculation model for the change component of the shaft alignment state is accurate for the deflection state of the shaft system.

Table 3. Pure translation and pure rotation RMSE table

\begin{tabular}{c|cc|cc|cc|c}
\hline No. & \multicolumn{2}{|c|}{$\# 1$} & \multicolumn{2}{c|}{$\# 2$} & \multicolumn{2}{c|}{$\# 3$} & $\# 4$ \\
\hline Error type & HOE & VOE & HOE & VOE & HOE & VOE & VAE \\
\hline RMSE result & $0.17 \%$ & $0.42 \%$ & $0.15 \%$ & $0.28 \%$ & $0.20 \%$ & $0.42 \%$ & $1.38 \%$ \\
\hline
\end{tabular}

Experiments \#5 and \#6 were carried out for parallel-rotating combined conditions. The experimental results (Figure 10) show that the offset errors are all less than $0.01 \mathrm{~mm}$. The vertical angularity errors are concentrated in the range of $-0.05 \sim 0.05 \mathrm{~mm} / \mathrm{m}$. The results of panning combined movement RMSE are shown in Table 4. Considering the parallel accuracy motion of the experimental platform $(0.01 \mathrm{~mm})$ and the rotational accuracy of the experimental platform $(0.0875 \mathrm{~mm} / \mathrm{m})$, for the shaft system deviation state, the calculation of the offset deflection of the centering state change component model is accurate

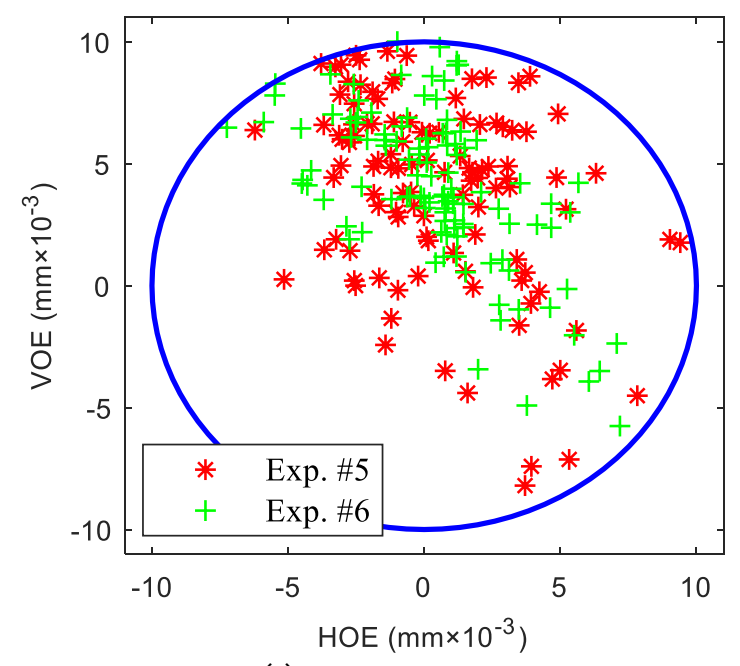

(a) Offset error graph

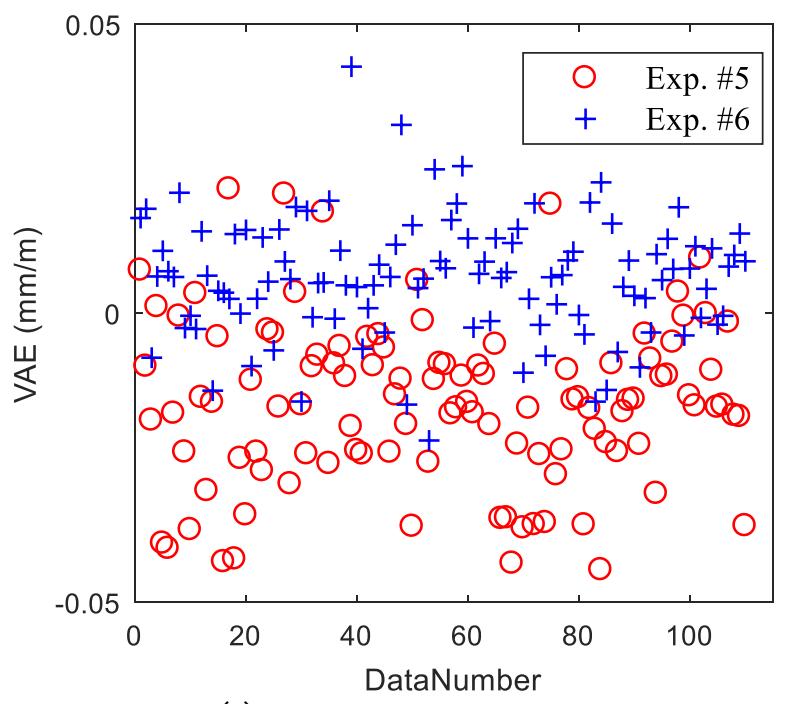

(b) Angularity error graph

Figure 10. The deviation error diagram of \#5 and \#6 experiment

Table 4. RMSE table of panning combined movement

\begin{tabular}{|c|c|c|c|c|c|c|}
\hline No. & \multicolumn{3}{|c|}{$\# 5$} & \multicolumn{3}{c|}{$\# 6$} \\
\hline Error type & HOE & VOE & VAE & HOE & VOE & VAE \\
\hline RMSE result & $0.31 \%$ & $0.55 \%$ & $2.10 \%$ & $0.28 \%$ & $0.52 \%$ & $1.19 \%$ \\
\hline
\end{tabular}

The above mentioned three types of experiments, namely, purely parallel motion conditions, purely rotational conditions and combined translational motion conditions, correspond to the three types of shaft alignment offset, deflection, and deviation respectively. The error of the centering component solution proposed in this paper and the actual maneuvering centering component is less than the accuracy of the translational motion of the experimental bench, and the centering component solution proposed in this paper is accurate.

\section{Conclusion}

Aiming at the problem of the change of in the alignment state caused by the deformation of the hull, a research on the online method of the propulsion shaft alignment monitoring adapted to the influence of the hull deformation has been carried out. Some important findings are as follows:

a) A new shafting alignment calculation model is established. Based on Euler's rigid body attitude theory, the shaft alignment is calculated by calculating the position-posture of the main shaft and the driven shaft 
b) A shafting alignment monitoring system adapted to the deformation of the hull is constructed. The laser displacement sensor fixed on the main shaft and driven shaft bracket monitors the shaft alignment status, which is suitable for situations which both the main and driven shafts may have position-posture changes.

c) The model has engineering application value and potential. It can be used in engineering to realize online shafting centering monitoring under the running state of the ship's propulsion shafting. Compensation control for the influence of hull deformation under different operating conditions of the ship is realized.

d) There are still some problems with the model in this paper. The shaft alignment model is constructed based on the assumption that the main shaft and driven shaft brackets do not undergo axial deformation and are approximated by small angles. Further research is needed to consider shaft deformation monitoring methods and large-angle issues.

\section{References}

1. Zhao Y. Cause Analysis of Doule Frequency Component Existing in Rotating Machinery Vibration Frequency Spectrum and Its Treatment[J](in Chinese). Inner Mongolia Electric Power, 2018.

2. Zw A, Jza B, Zja B, et al. Quantitative misalignment detection method for diesel engine based on the average of shaft vibreation and shaft shape characteristcs[J].Measurement,2021.

3. Qin W, Qin H, Zheng H, et al. The coupled effect of bearing misalignment and friction on vibration characteristics of a propulsion shafting system[J]. Proceedings of the Institution of Mechanical Engineers Part M Journal of Engineering for the Maritime Environment, 2017:147509021772287

4. Hujare D P, Karnik M G. Vibration responses of parallel misalignment in Al shaft rotor bearing system with rigid coupling[J]. Materials Today: Proceedings, 2018

5. ATHP, BAKD. Vibration response of misaligned rotors[J]. Journal of Sound and Vibration, 2009, 325( 3):609628.

6. Fu C, Yang Y, Lu K, et al. Non-linear vibration analysis of a rotor system with parallel and angular misalignments under uncertainty via a Legendre collocation approach[J]. International Journal of Mechanics and Materials in Design, 2019(2).

7. Simm Anthony, Wang Qing, Huang Songling, Zhao Wei. Laser based measurement for the monitoring of shaft misalignment[J].Measurement,2016,Vol.87: 104-116

8. Ruan X K, Yang P, Yan X P, et al. Study on hull Deformation by Integrated Model of Hull with Shaft[J]. Advanced Materials Research, 2014, 904:450-453.

9. Garg S, Kumar S, Deshpande R, et al. A Laser Shaft Alignment by IPS-PSD's for Rotary machine[J]. International Journal of Engineering and Technology, 2017, 4(4):6.

10. Li M, Wang X. The Design and Develop of A Laser Shaft Alignment Instrument Based on The Two Dimensional PSD[J]. International Journal of Intelligent Engineering \& Systems, 2012, 5(2):25-32.

11. Geng H C, Zheng S Y, Chen J P. Influence Analysis of Large Vessel Hull Deformation on Shafting Alignment[J](in Chinese). Ship Engineering, 2010.

12. Shi L, Xue D X, Song X G. Study of dynamic shafting alignment considering hull deformations[J](in Chinese). Journal of Dalian University of Technology, 2011.

13. Lei S, Xue D, Song X. Research on shafting alignment considering hull deformations $[J]$. Marine Structures, 2010, 23(1):103-114.

14. Zeyuan LI, Wang J, Liu Y. Shaft Dynamic Alignment Algorithm Considering Hull Deformation[J](in Chinese). Ship Engineering, 2018.

15. Seo C O, Jeong B, Kim J R, et al. Determining the influence of hull deformations caused by draught change on shaft alignment application using FE analysis[J]. Ocean Engineering, 2020, 210:107488.

16. Bu W J , He L, Shi L, et al. Output shaft alignment attitude real time monitoring for a marine main engine air mounting system[J](in Chinese). Journal of Vibration and Shock, 2009.

17. Shi L, Lin HE, Wei XU, et al. Main engine air suspension system alignment control algorithm[J](in Chinese). Journal of Vibration and Shock, 2010:1-5.

18. Wei XU, Lin HE, Shi L. Alignment disturbance control of an air-suspended main engine[J](in Chinese). Journal of Vibration and Shock, 2011.

19. Lin H E, Wei X U, Wen-Jun B U, et al. Development and application of new-type mounting system for naval vessel propulsion system[J](in Chinese). Journal of Ship Mechanics, 2013.

20. Yang Rui, Wang Zhuang, Li Xiaobin, Chen Wei, Wang Haojie. Development and trend of naval vessel floating raft vibration isolation system[J](in Chinese).Ship Engineering,2020,Vol.42(7): 28-34 
21. Zhang Le, Xu Wei, Li Zhengmin. Analysis of the influence of elastic support on ship shafting[J](in Chinese). Ship Science and Technology,2020,Vol.42(7): 81-84

22. Wen-Jun BU, Lin HE. Alignment control straegy for an air spring mounting system of marine propulsion engine[J](in Chinese). Journal of Vibration and Shock, 2012.

23. Lal M, Tiwari R. Experimental Estimation of Misalignment Effects in Rotor-Bearing-Coupling Systems[M]. Springer InternatioMaintenance Fundamentals (Second Edition), 2004:71-111.

24. Sinha J K, Lees A W, Friswell M I. Estimating unbalance and misalignment of a flexible rotating machine from a single run-down[J]. Journal of Sound \& Vibration, 2004, 272(3-5):967-989.

\section{Author contributions}

J.C. wrote the main manuscript text figures . All authors reviewed the manuscript.

Competing interests

The authors declare no competing interests.

\section{Additional information}

Correspondence and requests for materials should be addressed to W.B. 